Pacific Journal of Mathematics

ANTI-COMMUTATIVE ALGEBRAS AND HOMOGENEOUs 


\title{
ANTI-COMMUTATIVE ALGEBRAS AND HOMOGENEOUS SPACES WITH MULTIPLICATIONS
}

\author{
A. Sagle And J. SCHumi
}

As a generalization of certain results for Lie groups it is shown that an $n$-dimensional $H$-space $(M, \mu)$ with identity $e$ has a coordinate system at $e$ in which $\mu$ can be represented by a function $F: R^{n} \times R^{n} \rightarrow R^{n}$ which is analytic at $(0,0)$ and that the second derivative of $F$ induces a bilinear anti-commutative multiplication $\alpha$ on $R^{n}$. In this way an algebra $\left(R^{n}, \alpha\right)$ analogous to the Lie algebra of a Lie group is obtained and all such algebras are shown to be isomorphic. If $M=G / H$ is a reductive homogeneous space, then these results generalize the Lie group-Lie algebra correspondence and the algebra $\left(R^{n}, \alpha\right)$ induces a $G$-invariant connection on $G / H$. Relative to this connection it is shown that an automorphism of $(G / H, \mu)$ is an affine map and induces an algebra automorphism of $\left(R^{n}, \alpha\right)$. Also the connection is irreducible if $(G / H, \mu)$ has no proper invariant subsystems (the analog of normal subgroups). In the case where $G / H$ has a Riemannian structure, it may happen that there are no local isometries among the coordinate maps which give rise to anti-commutative multiplications on $R^{n}$.

1. Multiplications and change of coordinates. Let $M$ be an $n$-dimensional real, analytic manifold and let $\mu: M \times M \rightarrow M$ be an analytic function such that $\mu(e, e)=e$ for some $e \in M$. In this case $\mu$ is called a multiplication on $M$ and we denote this multiplicative structure by $(M, \mu)$. In the examples we consider, $e$ is a two-sided identity element; that is, $(M, \mu)$ is an $H$-space (for other examples see [6]). In particular we will consider Lie groups and Moufang loops $[\mathbf{1}, \mathbf{8}]$.

For the multiplicative structure $(M, \mu)$ let $(U, \phi)$ be a coordinate system at $e \in M$ where $U$ is a neighborhood of $\dot{e}$ and $\phi: U \rightarrow R^{n}$ is the coordinate map. Assume that $\phi(e)=0$ in $R^{n}$ and let $\phi^{-1}: U_{0} \rightarrow M$ denote the local inverse function of $\phi$ defined on a neighborhood $U_{0}$ of 0 . For $D \subset U_{0}$ a suitable neighborhood of $0 \in R^{n}$ we can represent $\mu$ in the coordinate system $\left(\phi^{-1}(D),\left.\phi\right|_{\phi^{-1}(D)}\right)$ as $\mu\left(\phi^{-1} X, \phi^{-1} Y\right)=\phi^{-1} F(X, Y)$ for $X, Y \in D$ where $F: D \times D \rightarrow U_{0}$ is analytic at $(0,0) \in D \times D$ and defines a "local multiplicative structure" $\left(U_{0}, F\right)$.

Let $\theta=(0,0)$; then since $F$ is analytic we can form the $k$ th derivative $F^{k}=F^{k}(\theta)$, which is a symmetric $k$-multilinear form on $R^{n}$ and, using the notation $F^{k} Z^{(k)}=F^{k}(Z, Z, \cdots, Z)$, with $Z=(X, Y)$, we can write 


$$
\begin{aligned}
F(X, Y)= & F(\theta)+F^{1}(X, Y)+\frac{1}{2} F^{2}(X, Y)^{(2)} \\
& +\sum_{k=3}^{\infty} \frac{1}{k !} F^{k}(X, Y)^{(k)} .
\end{aligned}
$$

Since $\mu(e, e)=e$, we obtain $F(0,0)=0$. Using the linearity of $F^{1}$ on $R^{n} \times R^{n}$, it follows that

$$
\begin{aligned}
F^{1}(X, Y) & =F^{1}((X, 0)+(0, Y)) \\
& =P X+Q Y
\end{aligned}
$$

where

$$
P X=F^{1}(X, 0)
$$

and

$$
Q Y=F^{1}(0, Y)
$$

Similarly, using the bilinearity of $F^{2}$, we have

$$
\begin{aligned}
F^{2}(X, Y)^{(2)} & =F^{2}((X, Y),(X, Y)) \\
& =F^{2}(X, 0)^{(2)}+2 F^{2}((X, 0),(0, Y))+F^{2}(0, Y)^{(2)}
\end{aligned}
$$

Next we assume that $(M, \mu)$ is an $H$-space (or more generally a local $H$-space) with $e$ the two-sided identity element. Then since $\mu(x, e)=x$, it follows that $F(X, 0)=X$ for all $X \in R^{n}$ sufficiently near 0 , which implies

$$
P X=X \quad \text { and } \quad F^{k}(X, 0)^{(k)}=0 \quad \text { for } \quad k=2,3, \cdots
$$

Similarly $\mu(e, x)=x$ implies

$$
Q X=X \quad \text { and } \quad F^{k}(0, X)^{(k)}=0 \quad \text { for } \quad k=2,3, \cdots .
$$

Thus the Taylor's series representing $\mu$ has the form

$$
F(X, Y)=X+Y+\alpha(X, Y)+\cdots
$$

where $\alpha(X, Y)=F^{2}((X, 0),(0, Y))$ defines a bilinear function $\alpha: R^{n} \times$ $R^{n} \rightarrow R^{n}$. That is, $R^{n}$ with the multiplication $\alpha$ becomes a nonassociative algebra which we denote by $\left(R^{n}, \alpha\right)$.

For example, let $G$ be an $n$-dimensional Lie group with Lie algebra $g$ and identify $g$ and $R^{n}$ as vector spaces. Then as above the Lie group multiplication $\mu$ induces the bilinear multiplication $\alpha$ on $g$ relative to some coordinate system $(U, \phi)$ at $e \in G$. Denoting this algebra by 
$(g, \alpha)$, we will show for $\phi^{1}=\phi^{\prime}(e)$, the differential of $\phi$ at $e$, that the original multiplication $[X, Y]$ in $g$ satisfies

$$
\phi^{\prime}[X, Y]=\alpha\left(\phi^{1} X, \phi^{1} Y\right)-\alpha\left(\phi^{1} Y, \phi^{1} X\right) .
$$

Thus the Lie algebra $g$ is isomorphic to the algebra $(g, \alpha)^{-}$which is the vector space $g$ with multiplication $\alpha(X, Y)-\alpha(Y, X)$; consequently the algebra $(g, \alpha)$ is Lie admissible [9]. The proof of the above formula is contained in Remark 3 below. However, if a canonical coordinate system is used, the Taylor's series representing $\mu$ is given by the Campbell-Hausdorff formula $X+Y+\frac{1}{2}[X, Y]+\cdots$; see [8]. So relative to a canonical coordinate system the nonassociative algebra induced on $g$ by $\mu$ has bilinear multiplication $\frac{1}{2}[X, Y]$. In particular, in the case of a Lie group there always exists a coordinate system in which the nonassociative algebra induced on $g$ by $\mu$ is anti-commutative. We will now prove that this is true in general for analytic $H$-spaces (or more generally, local analytic $H$-spaces).

Let $(M, \mu)$ be an analytic $H$-space with identity element $e$ and with coordinate system $(U, \phi)$ at $e$. As before, represent $\mu$ by

$$
\mu\left(\phi^{-1} X, \phi^{-1} Y\right)=\phi^{-1} F(X, Y)
$$

where $\phi^{-1}$ is the local inverse of $\phi$ and $F(X, Y)=$ $X+Y+\alpha(X, Y)+\cdots$. Now for a suitable neighborhood $W$ of $0 \in R^{n}$ we define a function $\psi: W \rightarrow R^{n}$, analytic at 0 in $R^{n}$, by the formula

$$
\psi(X)=X-\frac{1}{2} \alpha(X, X)
$$

Then since $(D \psi)(0)=I$, the inverse function theorem implies there is a neighborhood $V$ of 0 in $R^{n}$ so that $(V, \psi)$ is a coordinate system at 0 in $R^{n}$ and $\psi(0)=0$.

Next for $X, Y$ near 0 in $R^{n}$, define the function $K$ by

$$
K(X, Y)=\psi F\left(\psi^{-1} X, \psi^{-1} Y\right)
$$

Using (*), we see that the $R^{n}$-valued function $z=\psi \circ \phi$ restricted to a suitable neighborhood $U^{\prime}$ of $e$ gives a coordinate system in which $\mu$ is represented by

$$
\begin{aligned}
\mu\left(z^{-1} X, z^{-1} Y\right) & =\mu\left(\phi^{-1} \circ \psi^{-1} X, \phi^{-1} \circ \psi^{-1} Y\right) \\
& =\phi^{-1} F\left(\psi^{-1} X, \psi^{-1} Y\right) \\
& =\phi^{-1} \circ \psi^{-1} K(X, Y) \\
& =z^{-1} K(X, Y)
\end{aligned}
$$


for $X, Y$ near 0 in $R^{n}$. As in the previous consideration of $F$, note that $K$ has the Taylor's series

$$
K(X, Y)=X+Y+\beta(X, Y)+\cdots
$$

where $\beta: R^{n} \times R^{n} \rightarrow R^{n}$ is the bilinear term. Using the equation $\psi F(X, Y)=K(\psi X, \psi Y)$ and the series for $F, K$ and $\psi$, we observe that up to degree two the approximations are

$$
\begin{aligned}
\psi F(X, Y) & =F(X, Y)-\frac{1}{2} \alpha(F(X, Y), F(X, Y))+\cdots \\
& =X+Y-\frac{1}{2} \alpha(X, X)-\frac{1}{2} \alpha(Y, Y)+\frac{1}{2}[\alpha(X, Y)-\alpha(Y, X)]+\cdots
\end{aligned}
$$

and

$$
\begin{aligned}
K(\psi X, \psi Y) & =\psi X+\psi Y+\beta(\psi X, \psi Y)+\cdots \\
& =X+Y-\frac{1}{2} \alpha(X, X)-\frac{1}{2} \alpha(Y, Y)+\beta(X, Y)+\cdots
\end{aligned}
$$

From this we see

$$
\beta(X, Y)=\frac{1}{2}[\alpha(X, Y)-\alpha(Y, X)]
$$

Thus $\beta(X, Y)=-\beta(Y, X)$ and the algebra $\left(R^{n}, \beta\right)$ induced by $\mu$ relative to the coordinate system $\left(U^{\prime}, z\right)$ is anti-commutative.

REMARKS (1). The anti-commutative algebras induced by multiplications such as $\mu$ are unique up to isomorphism and consequently we call such an algebra the algebra associated with $\mu$. To see the isomorphism, let $(U, z)$ and $(\bar{U}, w)$ be coordinate systems at $e$ in which $\mu$ is represented by $\mu\left(z^{-1} X, z^{-1} Y\right)=z^{-1} K(X, Y)$ and by $\mu\left(w^{-1} X, w^{-1} Y\right)=w^{-1} \bar{K}(X, Y)$ as above. Let $K(X, Y)=X+Y+\beta(X, Y)+\cdots \quad$ and $\bar{K}(X, Y)=$ $X+Y+\bar{\beta}(X, Y)+\cdots$ with $\beta$ and $\bar{\beta}$ anti-commutative algebra multiplications on $R^{n}$. Next, note that the function $\eta=w \circ z^{-1}$ is analytic at 0 in $R^{n}$ with a series expansion about 0 given by $\eta(Z)=\eta^{1} Z+\frac{1}{2} \eta^{2} Z^{(2)}+\cdots$ for $Z$ sufficiently near 0 and that $\eta^{1}$ is nonsingular. From the above formulas for $\mu, K$ and $\bar{K}$ we have, for $X, Y$ sufficiently near 0 in $R^{n}$, that

$$
\begin{aligned}
\eta K(X, Y) & =w z^{-1} K(X, Y) \\
& =w \mu\left(z^{-1} X, z^{-1} Y\right) \\
& =w \mu\left(w^{-1}\left(w z^{-1} X\right), w^{-1}\left(w z^{-1} Y\right)\right) \\
& =w w^{-1} \bar{K}\left(w z^{-1} X, w z^{-1} Y\right) \\
& =\bar{K}(\eta X, \eta Y) .
\end{aligned}
$$


Now expanding $\eta, K, \bar{K}$ in their series, we obtain the 2nd degree approximations

$$
\begin{aligned}
\eta K(X, Y)= & \eta^{1} K(X, Y)+\frac{1}{2} \eta^{2} K(X, Y)^{(2)}+\cdots \\
= & \eta^{1} X+\eta^{1} Y+\eta^{1} \beta(X, Y)+\frac{1}{2} \eta^{2} X^{(2)} \\
& +\frac{1}{2} \eta^{2} Y^{(2)}+\eta^{2}(X, Y)+\cdots
\end{aligned}
$$

and

$$
\begin{aligned}
\bar{K}(\eta X, \eta Y)= & \eta X+\eta Y+\bar{\beta}(\eta X, \eta Y)+\cdots \\
= & \eta^{1} X+\eta^{1} Y+\frac{1}{2} \eta^{2} X^{(2)}+\frac{1}{2} \eta^{2} Y^{(2)} \\
& +\bar{\beta}\left(\eta^{1} X, \eta^{1} Y\right)+\cdots .
\end{aligned}
$$

These formulas imply

$$
\bar{\beta}\left(\eta^{1} X, \eta^{1} Y\right)-\eta^{1} \beta(X, Y)=\eta^{2}(X, Y) .
$$

Since $\beta$ and $\bar{\beta}$ are anti-commutative, the left side of this equation is skew-symmetric while $\eta^{2}$ is symmetric in $X$ and $Y$. Thus $\eta^{2}(X, Y)=0$, which implies $\eta^{1}$ is an isomorphism of the algebras $\left(R^{n}, \beta\right)$ and $\left(R^{n}, \bar{\beta}\right)$.

(2). The following observation will be needed in the next section. From formula (1), $\beta(X, Y)=\frac{1}{2}[\alpha(X, Y)-\alpha(Y, X)]$, we see that an automorphism of $\left(R^{n}, \alpha\right)$ is an automorphism of $\left(R^{n}, \beta\right)$.

We summarize some of these results as follows:

THEOREM 1. Let $(M, \mu)$ be an analytic $H$-space with identity element e. Then

(1) There exists a coordinate system $(U, z)$ at $e$ so that if $\mu$ is represented by $F(X, Y)=X+Y+\alpha(X, Y)+\cdots$, then the algebra $\left(R^{n}, \alpha\right)$ is anti-commutative and is unique up to isomorphism.

(2) The differential $\tau^{1}=\tau^{1}(e)$ of an analytic automorphism $\tau$ of $(M, \mu)$ induces an automorphism of $\left(R^{n}, \alpha\right)$.

To prove the last statement, let $\tau: M \rightarrow M$ be an analytic diffeomorphism with $\tau(e)=e$ and $\tau \mu(x, y)=\mu(\tau x, \tau y)$; that is, $\tau$ is an automorphism. Let $(U, z)$ be the coordinate system at $e$ given in Theorem 1 and let $z^{-1}: D \rightarrow M$ be a local inverse as before with $D$ a neighborhood of 0 in $R^{n}$. Since $\tau(e)=e$ and $z(e)=0$, we can write $\tau\left(z^{-1} X\right)=z^{-1} k(X)$ for $X$ near 0 in $R^{n}$, where $k$ is analytic at 0 and $k(0)=0$. Then for $X, Y$ near 0 in $R^{n}$ we have

$$
\begin{aligned}
\tau \mu\left(z^{-1} X, z^{-1} Y\right) & =\tau\left(z^{-1} F(X, Y)\right) \\
& =z^{-1}(k F(X, Y))
\end{aligned}
$$


and

$$
\begin{aligned}
\mu\left(\tau\left(z^{-1} X\right), \tau\left(z^{-1} Y\right)\right) & =\mu\left(z^{-1}(k X), z^{-1}(k Y)\right) \\
& =z^{-1}(F(k X, k Y)) .
\end{aligned}
$$

Since $\tau$ is an automorphism we obtain

$$
k(F(X, Y))=F(k(X), k(Y)) .
$$

Let $k$ have the Taylor's series

$$
k(X)=k^{1}(X)+\frac{1}{2} k^{2} X^{(2)}+\cdots
$$

where $X$ is near 0 in $R^{n}$ and $k^{m}=k^{m}(0)$ is the $m$ th derivative of $k$ at 0 . As in the computations in remark (1), we use the series for $F$ to obtain

$$
\alpha\left(k^{1} X, k^{1} Y\right)-k^{1} \alpha(X, Y)=k^{2}(X, Y)
$$

Since $\alpha$ is anti-commutative, we see that $k^{1} \alpha(X, Y)=\alpha\left(k^{1} X, k^{1} Y\right)$. Because $\tau$ is a diffeomorphism, we see that $k^{1}$ is nonsingular and therefore $k^{1}$ is an automorphism of $\left(R^{n}, \alpha\right)$.

REMARK (3). Modifying the notation of Remark 1, let $(U, z)$ be the coordinate system at $e \in M$ for which $\mu$ is represented by $\mu\left(z^{-1} X, z^{-1} Y\right)=z^{-1} K(X, Y)$ where $K(X, Y)=X+Y+\alpha(X, Y)+\cdots$ with $\alpha(X, Y)=-\alpha(Y, X)$. Next let $(\bar{U}, w)$ be any other coordinate system at $e$ for which $\mu$ is represented by $\mu\left(w^{-1} X, w^{-1} Y\right)=w^{-1} \bar{K}(X, Y)$ where $\bar{K}(X, Y)=X+Y+\bar{\beta}(X, Y)+\cdots$ with $\bar{\beta}$ bilinear. Then for $\eta=w \circ z^{-1}$, computations analogous to those in remark 1 yield $\eta K(X, Y)=\bar{K}(\eta X, \eta Y)$ and

$$
\bar{\beta}\left(\eta^{\prime} X, \eta^{\prime} Y\right)-\eta^{\prime} \alpha(X, Y)=\eta^{2}(X, Y) .
$$

Interchanging $X$ and $Y$ in this formula we obtain $\bar{\beta}\left(\eta^{1} Y, \eta^{1} X\right)-$ $\eta^{\prime} \alpha(Y, X)=\eta^{2}(Y, X)$. Subtracting these formulas and using the fact that $\eta^{2}$ is symmetric, we see that

$$
2 \eta^{\prime} \alpha(X, Y)=\bar{\beta}\left(\eta^{\prime} X, \eta^{\prime} Y\right)-\bar{\beta}\left(\eta^{\prime} Y, \eta^{\prime} X\right) .
$$

In particular, for a Lie group $G$ with $(U, z)$ a canonical coordinate system, we obtain the results previously mentioned concerning Lie 
admissible algebras. More generally, the above formula shows the algebra $\left(R^{n}, \alpha\right)$ is isomorphic to the algebra $\left(R^{n}, \frac{1}{2} \bar{\beta}\right)^{-}$which is the vector space $R^{n}$ with multiplication $\frac{1}{2}[\bar{\beta}(X, Y)-\bar{\beta}(Y, X)]$.

\section{Automorphisms and affine maps of a homogene-}

ous space. We apply the results of $\S 1$ to a homogeneous space with multiplication $\mu$ to obtain an invariant connection from the anticommutative algebra associated with $\mu$; see $[6,8]$. For certain homogeneous spaces we show that an automorphism of the multiplicative structure is an affine map of the corresponding connection.

Let $G$ be a connected Lie group with Lie algebra $g$ and let $H$ be a closed (Lie) subgroup with Lie algebra $h$. The pair $(G, H)$ or $(g, h)$ is called a reductive pair if there exists a subspace $m$ of $g$ such that $g=m+h$ (subspace direct sum) and $(\operatorname{Ad} H)(m) \subset m$; that is, in terms of algebras $[h, m] \subset m$. The corresponding analytic manifold $G / H$ is called a reductive homogeneous space. In most of the examples considered in [6] $G$ and $H$ are semi-simple with a decomposition $g=m+h$ where $m=h^{\perp}$ is the orthogonal complement relative to the Killing form of $g$.

For $\mathrm{G} / \mathrm{H}$ a reductive homogeneous space with a fixed decomposition $g=m+h$, Nomizu $[3,2]$ established a $1-1$ correspondence between $G$-invariant affine connections $\nabla$ on $G / H$ and nonassociative algebras $(m, \alpha)$ satisfying $\operatorname{Ad} H \subset \operatorname{Aut}(m, \alpha)$ where $\alpha: m \times m \rightarrow m$ is the algebra multiplication and $\operatorname{Aut}(m, \alpha)$ is the automorphism group of $(m, \alpha)$. On the algebra level, Ad $H \subset \operatorname{Aut}(m, \alpha)$ corresponds to ad $h \subset$ $D(m, \alpha)$, where $D(m, \alpha)$ is the Lie algebra of derivations of the algebra $(m, \alpha)$. For example, if $\nabla$ corresponds to the algebra $(m, \alpha)$, then for all $X \in m$ the one-parameter subgroups $\exp t X$ in $G$ project into geodesics (relative to $\nabla$ ) in $G / H$ by $\pi: G \rightarrow G / H$ if an only if $\alpha(X, Y)=$ $-\alpha(Y, X)$. Further, if $\nabla$ has zero torsion, then $\alpha(X, Y)=\frac{1}{2}[X, Y]_{m}$ where $[X, Y]_{m}$ is the projection of $[X, Y]$ in $g$ onto $m$; see $[3,8]$.

Next, let $M=G / H$ be a reductive space and let $(G / H, \mu)$ be an $H$-space as in $\S 1$ with $\bar{e}=e H$ the 2-sided identity; then we obtain an algebra $(m, \alpha)$ from $\mu$ relative to the canonical coordinate system obtained from $\pi \circ$ exp. For $u \in H$ let $\tau(u): G / H \rightarrow G / H: \bar{x} \rightarrow \overline{u x}$ and let $\tau(H)=\{\tau(u): u \in H\}$; then in [6] it was shown that $\tau(H) \subset$ $\operatorname{Aut}(G / H, \mu)$ implies $\operatorname{Ad} H \subset \operatorname{Aut}(m, \alpha)$ where $\operatorname{Aut}(G / H, \mu)$ is the automorphism group of $(G / H, \mu)$. Thus a multiplicative system $(G / H, \mu)$ with $\tau(H) \subset \operatorname{Aut}(G / H, \mu)$ induces a $G$-invariant connection on $G / H$ via the algebra $(m, \alpha)$. But from $\S 1$, there is a change of coordinates which determines an anti-commutative algebra $(m, \beta)$ which is unique up to isomorphism and is given by $2 \beta(X, Y)=$ $\alpha(X, Y)-\alpha(Y, X) . \quad$ By Remark (2), Ad $H \subset$ Aut $(m, \alpha)$ implies Ad $H \subset$ Aut $(m, \beta)$ and therefore the anti-commutative algebra $(m, \beta)$ gives rise to a $G$-invariant connection called the connection induced by $\mu$. Many 
examples are given in [6] and the Moufang Loop $S^{7}$ obtained from the Cayley numbers of norm 1 is discussed in [7].

REMARK (4). For a Lie group $(G, \mu)$ with associative multiplication $\mu$, the $G$-invariant connections are given by all the possible nonassociative algebras $(g, \alpha)$. However, these algebras need not arise from a fixed algebra $\left(g, \alpha_{0}\right)$ by using the formulas obtained from a change of coordinates at $e \in G$. For, as in Remark 3, any algebra $(g, \beta)$ which arises from a change of coordinates at $e$ in $G$ is Lie admissible with $(g, \beta)^{-}$isomorphic to the Lie algebra $g$. But there are many nonassociative algebras $(g, \alpha)$ which are not Lie admissible and consequently cannot be obtained via a change of coordinates.

We will now consider certain $H$-spaces $(G / H, \mu)$ which have properties analogous to Lie groups and the Moufang loop $S^{7}$. Thus we first assume $(G / H, \mu)$ is an analytic loop; that is, the left and right multiplications

$$
L(\bar{x}): G / H \rightarrow G / H: \bar{y} \rightarrow \mu(\bar{x}, \bar{y}) \text { and } R(\bar{x}): G / H \rightarrow G / H: \bar{y} \rightarrow \mu(\bar{y}, \bar{x})
$$

are analytic diffeomorphisms for all $\bar{x} \in G / H$. Next we observe that the set of all diffeomorphisms $L(\bar{x})$ and $R(\bar{y})$ of the loop $(G / H, \mu)$ generates a subgroup $\Gamma$ of the group of all diffeomorphisms. In particular note that a Lie group $G$ can be represented by the Lie group $K$ generated by all the maps $L(x)$. Also, the Moufang loop $S^{7}$ can be represented as a reductive space $K / H$ where $K \subset \Gamma$ is the Lie group generated by the maps $R\left(x^{2}\right) L(x)$ for all $x \in S^{7}$ and $\tau(H)$ is contained in the automorphism group of $S^{7}$; see [7]. Using this notation we have the following definition.

Definition. An analytic loop $(M, \mu)$ is called multiplicatively homogeneous if in the group $\Gamma$ generated by all the diffeomorphisms $L(x)$ and $R(y)$ for $x, y \in M$ there exists a Lie group $K \subset \Gamma$ satisfying:

(1) $K$ acts transitively on $M$, and

(2) $K$ is generated by a set of fixed monomial expressions in the functions $L(x)$ and $R(y)$ for all $x, y \in M$.

We now consider the relationship between automorphisms of a loop $(M, \mu)$ and affine maps of a connection $\nabla$ on $M$ which generalizes some well known results on Lie groups and Moufang loops. An affine map of a manifold $M$ with connection $\nabla$ is a diffeomorphism $f: M \rightarrow M$ such that $f^{\prime} \nabla(X, Y)=\nabla\left(f^{\prime} X, f^{\prime} Y\right)$ for all vector fields $X, Y$ on $M$ where $f^{\prime}$ is the differential of $f$.

THEOREM 2. Let $(M, \mu)$ be a multiplicatively homogeneous analytic loop such that $M$ can be represented as a reductive homogeneous space 
$K / H$ with $K$ as above and $\tau(H) \subset \operatorname{Aut}(K / H, \mu)$. Then an analytic automorphism of $(K / H, \mu)$ is an affine map relative to the invariant connection induced by $\mu$.

Proof. Since $(K, H)$ is a reductive pair we have a Lie algebra decomposition $k=m+h$ and from Theorem 1 the differential $f^{\prime}=f^{\prime}(\bar{e})$ of an automorphism $f \in \operatorname{Aut}(K / H, \mu)$ is an automorphism of the algebra $(m, \beta)$ associated with $\mu$.

Next note that $f$ being an automorphism of $(K / H, \mu)$ implies

$$
f L(\bar{x}) f^{-1}=L(f \bar{x}) \quad \text { and } \quad f R(\bar{y}) f^{-1}=R(f \bar{y})
$$

for all $\bar{x}, \bar{y} \in K / H$. Thus if $k=m\left(L\left(\bar{x}_{1}\right), R\left(\bar{y}_{1}\right), \cdots\right) \in K$ is a monomial generator expression, we see that $f k f^{-1}=m\left(L\left(f \bar{x}_{1}\right), R\left(f \bar{y}_{1}\right), \cdots\right)$ is in $K$. Consequently

$$
f \tau(K) f^{-1} \subset \tau(K)
$$

where for any $a \in K$ we have $\tau(a): K / H \rightarrow K / H: \bar{x} \rightarrow \overline{a x}$ and $\tau(K)=$ $\{\tau(a): a \in K\}$. Thus for any $a \in K$, there exists $a^{\prime} \in K$ such that

$$
f \tau(a) f^{-1}=\tau\left(a^{\prime}\right)
$$

and this implies $f$ locally commutes with $K$ as defined in [4]. It is also shown in [4] that if $\phi$ is an analytic diffeomorphism of $K / H$ with $\phi(\bar{e})=\bar{e}$ such that $\phi$ locally commutes with $K$ and $\phi^{\prime} \in \operatorname{Aut}(m, \beta)$, then $\phi$ is an affine map of $K / H$ relative to the connection given by $(m, \beta)$. This result, along with the fact that $f^{\prime} \in \operatorname{Aut}(m, \beta)$, proves $f$ is an affine map.

REMARK (5). In the above proof the restrictions on $K$ were used to show $f \tau(K) f^{-1} \subset \tau(K)$, which was needed to prove the local commuting property; thus the preceding proof can be generalized to give the following result.

Corollary 3. Let $(G, H)$ be a reductive pair and let $(G / H, \mu)$ be an $H$-space with identity $\bar{e}$ such that $\tau(H) \subset$ Aut $(G / H, \mu)$. Let $f$ be an analytic automorphism of $(G / H, \mu)$, so that $f(\bar{e})=\bar{e}$ and $f \tau(G) f^{-1} \subset \tau(G)$. Then $f$ is an affine map of $G / H$ relative to the connection induced by $\mu$.

\section{Normal subsystems and holonomy reducibility.} For an analytic $H$-space $(M, \mu)$ we now define local inverses and show how they can be used to generalize the concept of a normal subgroup of a Lie group. We then observe the relation between 
these subsystems and the irreducibility of the connection on a reductive space $M=G / H$ induced by $\mu$.

Let the $H$-space $(M, \mu)$ have identity $e$ and, relative to a suitable coordinate system $(U, \phi)$ at $e$ with $\phi(e)=0$ in $R^{n}$, let $\mu$ be represented by

$$
F(X, Y)=X+Y+\alpha(X, Y)+\cdots
$$

At $\theta=(0,0) \in R^{n} \times R^{n}$, the partial derivative of $F$ relative to the second variable is given by $\left(D_{2} F\right)(\theta)(0, Y)=Y$ and thus the transformation $I=\left(D_{2} F\right)(\theta): R^{n} \rightarrow R^{n}$ is nonsingular. Therefore, by the implicit function theorem, there exists an open ball $B$ in $R^{n}$ with center at $0 \in R^{n}$ and a uniquely determined analytic map $r: B \rightarrow R^{n}$ such that $r(0)=0$ and $F(X, r(X))=0$ for all $X \in B$. These facts imply that there exists a neighborhood $V$ of $e$ in $M$ and a unique analytic function $\rho: V \rightarrow M$ such that $\rho(e)=e$ and $\mu(x, \rho(x))=e$ for all $x \in V$. Thus $(M, \mu)$ has a local right inverse function $\rho$ and similarly a local left inverse function.

Now assume that in the coordinate system in which $\mu$ is represented by $F(X, Y)$ the algebra $\left(R^{n}, \alpha\right)$ is anti-commutative as in Theorem 1. Then the local right inverse function $r$ has a series expansion

$$
r(X)=r^{1} X+\frac{r^{2}}{2} X^{(2)}+\cdots
$$

for $X$ near 0 and $r^{k}=r^{k}(0)$. This gives

$$
\begin{aligned}
0 & =F(X, r(X)) \\
& =X+r^{1} X+\frac{r^{2}}{2} X^{(2)}+\alpha\left(X, r^{1} X\right)+\cdots,
\end{aligned}
$$

which implies the approximation

$$
\begin{aligned}
r(X) & =-X+\alpha(X, X)+\epsilon(3) \\
& =-X+\epsilon(3)
\end{aligned}
$$

since $\alpha(X, X)=0$.

Definition. Let the $H$-space $(M, \mu)$ have identity $e$ and local right inverse function $\rho$. Then a submanifold $N$ of $M$ containing $e$ is called a locally invariant subsystem if $\mu(N, N) \subset N$ and there is neighborhood $U$ of $e$ in the domain of $\rho$ such that $\mu(\mu(x, y), \rho(x)) \in N$ whenever $x \in U$ and $y \in N$. 
REMARK (6). Let $N$ be a locally invariant subsystem of the $H$ space $(M, \mu)$ and identify the tangent space $T(N, e)$ with a vector subspace $n \subset R^{n}$. Then $(n, \alpha)$ is an ideal of the algebra $\left(R^{n}, \alpha\right)$ associated with $\mu$. To see this, let $\mu$ be represented by $F(X, Y)$ as before; then for $X \in R^{n}, Y \in n$ sufficiently near $0 \in R^{n}$, the local invariance of $N$ implies that $F(F(X, Y), r(X))$ is in $n$. Expanding the Taylor's series, we see that

$$
\begin{aligned}
F(F(X, Y), r(X)) & =F(X, Y)+r(X)+\alpha(F(X, Y), r(X))+\cdots \\
& =Y+2 \alpha(X, Y)+\epsilon(3)
\end{aligned}
$$

is in $n$. Since $Y \in n$, this implies $\alpha(X, Y) \in n$ and also $\alpha(Y, X)=$ $-\alpha(X, Y) \in n$; that is, $n$ is an ideal of $\left(R^{n}, \alpha\right)$.

We now let $M=G / H$ be a reductive homogeneous space and consider what a locally invariant subsystem implies about the holonomic properties of the induced connection; see $[\mathbf{2}, \mathbf{3}, \mathbf{4}, \mathbf{5}]$ for more results on holonomy. For the reductive pair $(G, H)$ with a fixed Lie algebra decomposition $g=m+h$ and $(\operatorname{Ad} H) m \subset m$, let the algebra $(m, \alpha)$ determine a $G$-invariant connection $\nabla$ as before. For $X, Y, Z \in m$ we have the map

$$
R(X, Y): m \rightarrow m: Z \rightarrow R(X, Y) Z
$$

where

$$
R(X, Y) Z=\alpha(X, \alpha(Y, Z))-\alpha(Y, \alpha(X, Z))-\alpha(X Y, Z)-[h(X, Y), Z]
$$

is the curvature of $\nabla$ evaluated at $\bar{e}=e H$ in $G / H$; recall that $X Y=$ $[X, Y]_{m}\left(\right.$ resp. $\left.h(X, Y)=[X, Y]_{h}\right)$ is the projection of $[X, Y]$ in $g$ onto $m$ (resp. $h$ ). The holonomy algebra of $G / H$ is the Lie algebra of the holonomy group of $G / H$ relative to $\nabla$. From $[2,3]$, we know that the holonomy algebra is the smallest Lie algebra $h^{*}$ of endomorphisms of $m$ such that $R(X, Y) \in h^{*}$ and $\left[L(X), h^{*}\right] \subset h^{*}$ for all $X, Y \in m$ where $L(X): m \rightarrow m: Y \rightarrow \alpha(X, Y)$. Denote $h^{*}$ by hol $(\alpha)$.

Remark (7). Let $L(m, \alpha)$ be the Lie algebra of endomorphisms generated by the set of all $L(X)$ for $X \in m$ and let $D(m, \alpha)$ be the Lie algebra of derivations of the algebra $(m, \alpha)$ which we now assume to be anti-commutative. Since the mappings ad $U: m \rightarrow m: X \rightarrow[U X]$ for $U \in h$ are in $D(m, \alpha)$, we see from the formulas for hol $(\alpha)$ that $\operatorname{hol}(\alpha) \subset L(m, \alpha)+D(m, \alpha)$ which is a Lie algebra since $[L(m, \alpha), D(m, \alpha)] \subset L(m, \alpha)$. We say that the holonomy group acts irreducibly on $G / H$ if $h o l(\alpha)$ acts irreducibly on $m$. The relation between irreducibility and the algebra $(m, \alpha)$ is as follows: Let $n$ be a 
proper ideal of the algebra $(m, \alpha)$; then in [4] it was shown that there exists a proper ideal $n^{\prime}$ of $(m, \alpha)$ which is $D(m, \alpha)$-invariant. Thus $\operatorname{hol}(\alpha) n^{\prime} \subset[L(m, \alpha)+D(m, \alpha)]\left(n^{\prime}\right) \subset n^{\prime}$ and therefore the action of hol $(\alpha)$ is reducible on $m$ if $(m, \alpha)$ has a proper ideal. We use the terminology that a locally invariant subsystem $N$ of $M$ is "proper" if its tangent space $n$ is a proper subspace of the tangent space of $M$. The proof of the following result now follows from remarks (7) and (8).

THEOREM 4. Let $(G / H)$ be a reductive pair with decomposition $g=m+h$ and let the $H$-space $(G / H, \mu)$ with identity $\bar{e}$ satisfy $\tau(H) \subset$ Aut $(G / H, \mu)$. If $(G / H, \mu)$ has a "proper" locally invariant subsysiem $N$, then the algebra $(m, \alpha)$ associated with $\mu$ has a proper ideal $n$ ' such that ad $h\left(n^{\prime}\right) \subset n^{\prime}$. Thus, in this case, $G / H$ is holonomy reducible relative to the connection induced by $\mu$.

REMARK (8). Let $(M, \mu)$ and $\left(M^{\prime}, \mu^{\prime}\right)$ be analytic $H$-spaces and let $\phi: M \rightarrow M^{\prime}$ be an analytic homomorphism of $M$ onto $M^{\prime}$. Then, as for Lie groups, the kernel of $\phi$ is a subsystem of $(M, \mu)$ which is also a locally invariant subsytem. Thus if $\phi$ is an analytic homomorphism of $(G / H, \mu)$ such that the kernel of $\phi$ is a "proper" invariant subsystem, then one obtains a proper ideal of the algebra $(m, \alpha)$ associated with $\mu$. Consequently $G / H$ is holonomy reducible relative to the connection induced by $\mu$. The converse-type statements appear to be false unless further associativity assumptions on $\mu$ are assumed.

4. Isometric change of coordinates. In $\S 1$ we showed that for an analytic $H$-space $(M, \mu)$ there exists a coordinate system in which the algebra $\left(R^{n}, \alpha\right)$ associated with $\mu$ is anti-commutative. However, if further conditions are imposed on the coordinates, then this need not be the case. In particular we shall now consider pseudoRiemannian connections and coordinates.

Let $G / H$ be a reductive homogeneous space with the usual decomposition $g=m+h$ and let $C^{*}$ be a pseudo-Riemannian metric $[2,8]$ which induces the $G$-invariant connection $\nabla$ corresponding to the algebra $(m, \alpha)$. Then $C^{*}$ is given by a symmetric nondegenerate form $C$ on $m$ such that for all $X, Y, Z \in m$ and $V \in h$ the following conditions are satisfied:

$$
\begin{aligned}
& C(\alpha(Z, X), Y)+C(X, \alpha(Z, Y))=0 \text { and } \\
& C((\operatorname{ad} V) X, Y)+C(X,(\operatorname{ad} V) Y)=0 .
\end{aligned}
$$

We denote such an algebra by $(m, \alpha, C)$; see $[\mathbf{5 , 1 0 ]}$ for more details. The algebra multiplication $\alpha$ is given uniquely by 


$$
\alpha(X, Y)=1 / 2 X Y+U(X, Y)
$$

where $X Y=[X, Y]_{m}$ as before, and $U(X, Y)=U(Y, X)$ is uniquely determined by

$$
2 C(U(X, Y), Z)=C(Z X, Y)+C(X, Z Y)
$$

Now suppose $D^{*}$ is another pseudo-Riemannian structure on $G / H$ which is given by a symmetric nondegenerate form $D$ on $m$. A mapping $f: m \rightarrow m$ with $f(0)=0$ is a local isometry relative to the structures $C$ and $D$ on $m$ if $f$ is a local diffeomorphism at 0 in $m$ and for $f^{1}=f^{1}(0)$ we have as usual $C\left(f^{1} X, f^{1} Y\right)=D(X, Y)$. With these formulas we prove the following results about a local isometric change of coordinates for an $H$-space $(G / H, \mu)$.

THEOREM 5. Let $M=G / H$ be a reductive homogeneous space with fixed Lie algebra decomposition $g=m+h$ and pseudoRiemannian structures $C^{*}$ and $D^{*}$. Let the algebras $(m, \alpha, C)$ and $(m, \beta, D)$ be obtained from the $H$-space multiplication $\mu$ on $G / H$ by coordinate maps $\phi_{1}$ and $\phi_{2}$ as before, and assume these algebras determine $G$-invariant pseudo-Riemannian connections relative to $C^{*}$ and $D^{*}$ respectively. If the change of coordinates map $\phi=\phi_{1}^{\circ} \phi_{2}^{-1}: m \rightarrow m$ is a local isometry, then the algebras $(m, \alpha, C)$ and $(m, \beta, D)$ are isomorphic.

In this case the new algebra is anti-commutative if and only if the original algebra is anti-commutative. Conditions for the algebra $(m, \alpha, C)$ inducing an invariant pseudo-Riemannian connection to be anti-commutative are discussed in [11]; roughly the conditions are that the algebra $(m, \alpha, C)$ must be power-associative.

For the proof first note that we have the following diagram:

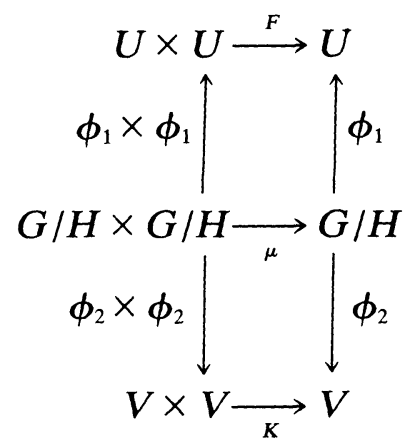

where $U$ and $V$ are suitable neighborhoods of 0 in $m$ and for $\phi=$ $\phi_{1}^{\circ} \phi_{2}^{-1}$ we have $F(\phi X, \phi Y)=\phi K(X, Y)$ for $X, Y$ near 0 in $m$. From the Taylor's series expansions of $\phi, F$ and $K$ we obtain as before 


$$
\alpha\left(\phi^{1} X, \phi^{1} Y\right)-\phi^{1} \beta(X, Y)=\phi^{2}(X, Y)
$$

where $\phi^{1}=\phi^{1}(0)$ and $\phi^{2}=\phi^{2}(0)$. Also using the fact that $\phi$ is a local isometry, we have

$$
C\left(\phi^{1} X, \phi^{1} Y\right)=D(X, Y) .
$$

Now $\beta$ satisfies formulas similar to those for $\alpha$; that is, $\beta$ is given by

$$
\beta(X, Y)=1 / 2 X Y+\tilde{U}(X, Y)
$$

where $\bar{U}(X, Y)=\bar{U}(Y, X)$ is uniquely determined by

$$
2 D(\bar{U}(X, Y), Z)=D(Z X, Y)+D(X, Z Y)
$$

Hence, we see from (3) that

$$
1 / 2 \phi^{1} X \phi^{1} Y+U\left(\phi^{1} X, \phi^{1} Y\right)-1 / 2 \phi^{1}(X Y)-\phi^{1} \bar{U}(X, Y)=\phi^{2}(X, Y) .
$$

Since $U, \bar{U}$ and $\phi^{2}$ are symmetric in $X$ and $Y$,

$$
U\left(\phi^{1} X, \phi^{1} Y\right)-\phi^{1} \bar{U}(X, Y)=\phi^{2}(X, Y) \text { and }
$$

$$
\phi^{1}(X Y)=\phi^{1} X \phi^{1} Y \text {. }
$$

Using equations (2), (4), (5) and (6) we see that

$$
\begin{aligned}
2 C( & \left.U\left(\phi^{1} X, \phi^{1} Y\right), \phi^{1} Z\right) \\
& =C\left(\phi^{1} Z \phi^{1} X, \phi^{1} Y\right)+C\left(\phi^{1} X, \phi^{1} Z \phi^{1} Y\right) \\
& =C\left(\phi^{1}(Z X), \phi^{1} Y\right)+C\left(\phi^{1} X, \phi^{1}(Z Y)\right) \\
& =D(Z X, Y)+D(X, Z Y) \\
& =2 D(\bar{U}(X, Y), Z) \\
& =2 C\left(\phi^{1} \bar{U}(X, Y), \phi^{1} Z\right) .
\end{aligned}
$$

Since $C$ is nondegenerate and $\phi^{1}$ is nonsingular, we obtain

$$
\phi^{1} \bar{U}(X, Y)=U\left(\phi^{1} X, \phi^{1} Y\right)
$$

Thus from the formulas for $\alpha, \beta$ and (6) we see $(m, \alpha, C)$ and $(m, \beta, D)$ are isomorphic; this proves Theorem 5 . 
REMARK (9). The above result shows an isometry induces an isomorphism of algebras. However the results in [4] indicate the converse is false in general; the local commuting property is needed.

\section{REFERENCES}

1. R. H. Bruck, Survey of Binary Systems, Springer-Verlag, Berlin, 1958.

2. S. Kobayashi and K. Nomizu, Foundations of Differential Geometry, I and II, John Wiley Inc., New York, 1963 and 1968.

3. K. Nomizu, Invariant affine connections on homogeneous spaces, Amer. Math. J., 76 (1954), 33-65.

4. A. Sagle, On anti-commutative algebras and homogeneous spaces, J. Math. and Mech., 16 (1967), 1381-1394.

5. A. Sagle, On homogeneous spaces, holonomy and nonassociative algebras, Nagoya Math. J., 32 (1968), 373-395.

6. A. Sagle and J. Schumi, Multiplications on homogeneous spaces, nonassociative algebras and connections, Pacific J. Math., 48 (1973), 247-265.

7. Connections on $S^{7}$, to appear.

8. A. Sagle and R. Walde, Introduction to Lie Groups and Lie Algebras, Academic Press, New York, 1973.

9. A. Sagle, On reductive Lie admissible algebras, Canad. J. Math., 23 (1971), 325-331.

10. Jordan algebras and connections on homogeneous spaces, Trans. Amer. Math. Soc., 187 (1974), 405-427.

11. - Power-associative algebras and Riemannian connections, Pacific J. Math., 65 (1976), $493-498$

Received June 26, 1976 and in revised form August 17, 1976. The authors thank the referee for his suggestions.

UNIVERSITY OF HaWAII AT HILO,

Hilo, HaWAII 96720

AND

St. Paul Fire and Marine Ins. Co.,

St. Paul, Minnesota 




\section{Pacific Journal of Mathematics}

Vol. 68, No. 1

March, 1977

Richard Julian Bagby, On $L^{p}, L^{q}$ multipliers of Fourier transforms . .......

Robert Beauwens and Jean-Jacques Van Binnebeek, Convergence theorems in

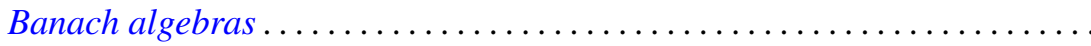

James Cyril Becker, Skew linear vector fields on spheres in the stable

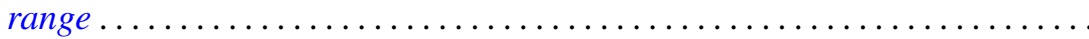

Michael James Beeson, Continuity and comprehension in intuitionistic formal

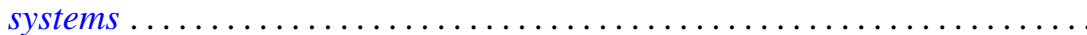

James K. Deveney, Generalized primitive elements for transcendental field

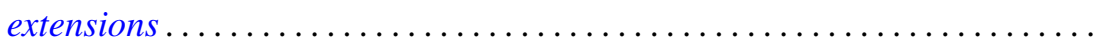

Samuel S. Feder, Samuel Carlos Gitler and K. Y. Lam, Composition properties

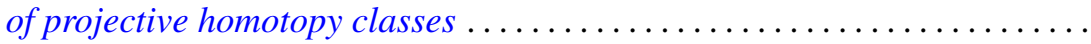

Nathan Jacob Fine, Tensor products of function rings under composition ......

Benno Fuchssteiner, Iterations and fixpoints . . . . . . . . . . . . . .

Wolfgang H. Heil, On punctured balls in manifolds

Shigeru Itoh, A random fixed point theorem for a multivalued contraction

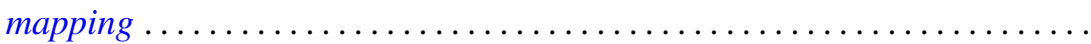

Nicolas P. Jewell, Continuity of module and higher derivations . . . . . . ......

Roger Dale Konyndyk, Residually central wreath products . . . . . . . . . . .

Linda M. Lesniak and John A. Roberts, On Ramsey theory and graphical

parameters.

Vo Thanh Liem, Some cellular subsets of the spheres.

Dieter Lutz, A perturbation theorem for spectral operators

P. H. Maserick, Moments of measures on convex bodies ... . . .

Stephen Joseph McAdam, Unmixed 2-dimensional local domains . .

D. B. McAlister and Norman R. Reilly, E-unitary covers for inverse semigroups...

William H. Meeks, III and Julie Patrusky, Representing codimension-one

homology classes by embedded submanifolds . . .

Premalata Mohapatro, Generalised quasi-Nörlund summability . .

Takahiko Nakazi, Superalgebras of weak-*Dirichlet algebras .

Catherine Louise Olsen, Norms of compact perturbations of operators .

William Henry Ruckle, Absolutely divergent series and isomorphism of

subspaces. II.

Bernard Russo, On the Hausdorff-Young theorem for integral operators .

Arthur Argyle Sagle and J. R. Schumi, Anti-commutative algebras and

homogeneous spaces with multiplications ............

Robert Evert Stong, Stiefel-Whitney classes of manifolds .

D. Suryanarayana, On a theorem of Apostol concerning Möbius functions of

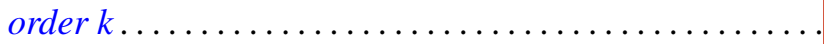

Yoshio Tanaka, On closedness of $C$ - and $C^{*}$-embeddings . . 